
\title{
25 Research Suare \\ Yoga Versus Physical Therapy In Multiple Sclerosis: Randomized Controlled Trial
}

\section{Elena Lysogorskaia ( $\square$ Kutakovaev@gmail.com )}

April Rehabilitation Center

\section{Timur Ivanov}

April Rehabilitation Center

Elena Ulmasbaeva

Yoga Practika LTD

\section{Aynagul Mendalieva}

April Rehabilitation Center

Maxim Youshko

Yoga Practika LTD

\section{Lev Brylev}

Bujanov Moscow City Clinical Hospital

\section{Research Article}

Keywords: yoga, lyengar method, physical therapy, exercise, multiple sclerosis, randomized controlled trial

Posted Date: December 28th, 2021

DOI: https://doi.org/10.21203/rs.3.rs-1123951/v1

License: (c) (1) This work is licensed under a Creative Commons Attribution 4.0 International License.

Read Full License 


\section{Abstract}

Background. Yoga originated in the territory of modern India more than 3000 years ago and uses techniques for working with the musculoskeletal system, cardiorespiratory system and attention. Currently, the effectiveness and safety of yoga in patients with various neurological disorders, including MS, is of interest to many scientists and clinicians. The main aim of this study is to examine the effect of yoga on symptoms and quality of life in patients with MS versus physical therapy (exercise therapy) and no exercise.

Methods. The patients were randomly assigned to 3 groups (yoga, PT, or waiting list), patients from the waiting list had an opportunity to enter the yoga program after the end of the trial period. After 12 weeks of regular exercises (or absence of them), the effect of yoga and PT on the functional status and quality of life of patients were evaluated. The MS treatment was a part of routine practice, as prescribed by the treating neurologist. The data was collected during the patients' two visits to the study center - before the start of the study and at the end of the 12-week period. The in-person examination included a doctor's assessment of the EDSS, the SF-36 quality of life questionnaire, the fatigue scale, the Berg balance scale, the 6-minute walking test.

Results. A total of 36 patients finished the clinical study: 30 women and 6 men. There was no statistically significant difference between the groups in terms of improvement in MS symptoms as measured by the balance, walking test and fatigue scales. However, in the analysis of the 8 criteria of SF-36 quality-of-life questionnaire the covariation analysis statistically significant differences were found in favor of the yoga group in terms of physical functioning (PF) ( $p=0.003)$, life activity (VT) $(p<0.001)$, mental health $(\mathrm{MH})(\mathrm{p}=013)$, social functioning $(\mathrm{SF})(\mathrm{p}=0.028)$.

Conclusions. Thus, regular yoga classes under the guidance of qualified staff are a promising method of non-drug rehabilitation of patients with MS with motor disorders. More research is needed to examine the impact of yoga on clinical patient improvement and quality of life indicators.

\section{Trial registration. ISRCTN15486200 https://doi.org/10.1186/ISRCTN15486200}

\section{Background}

Multiple sclerosis (MS) is a progressive, chronic disease of the central nervous system, which manifests itself in various neurological symptoms and their combinations, debuts more often at a young age, and may have different variants of the course. As a result, patients experience temporary or progressive disturbances of movement, walking function, balance, restriction of self-reliance, and a decrease in quality of life. The most common symptoms of multiple sclerosis are muscle weakness and instability in walking, spasticity, pathological fatigue, and other symptoms of CNS lesions may occur at various levels (Scierlo S. 2017). In this regard, MS is one of the frequent reasons for the increased disability length of different age groups of patients. Studies show that more than $65 \%$ of patients have trouble moving and up to $85 \%$ of patients have gait disorders (Rogers K. 2015). Thus, all MS patients need comprehensive 
rehabilitation and for many patients, it is required in the early stages of the disease. There is evidence that physical therapy programs (physical exercises) can improve MS patients' functionality (mobility, walking, endurance) and quality of life (Khan T. 2007, Alphonsus K. 2019, Motl RW. 2020). There is also evidence that more than $60 \%$ of patients are willing to use additional approaches to drug treatment, which can include exercise (Miller P. 2017). Among the actively studied methods of physical therapy used in the rehabilitation of patients with MS, there is physical therapy, yoga classes, dance therapy, aerobic exercises, and a combination of these approaches.

The effectiveness of physical therapy has beenexamined in many studies. A review of 26 randomized trials found a reduction in the risk of aggravation in the group of patients with MS performing exercise compared to the patients without gymnastics (6.3\% vs 4.6\%), i and additionally demonstrated the safety of exercise (Pilutti LA. 2014 ). Thus, exercises can influence the course of the disease in patients with MS. There is also widely presented in the literature information about the effectiveness of exercise to reduce specific symptoms of MS (Wens I. 2015, Razazian N. 2016). Thus, 45 researches were conducted to study the effects of 69 different types of exercise in 2,250 patients with pathological fatigue in MS (Heine M. 2015). These researches demonstrate the effectiveness of exercise to control the fatigue of patients with multiple sclerosis.

any studies, as well as in the meta-analysis, have obtained data on the effectiveness of yoga in patients with MS on reducing the level of depression, pain, pathological fatigue, bladder dysfunction, postural disorders, and improvement in the quality of life and mobility (Patil N. 2012, Salgado B. 2013, Karbandi S. 2015, Rogers K. 2015, Chobe S. 2016). The safety and efficiency of yoga compared to the non-exercise group of patients are demonstrated in a variety of studies ranging in duration from eight weeks to six months (Cohen E., 2017, Kahraman T. 2018, Nejati S, 2016).

Despite a large number of positive results of using yoga and physical therapy for patients with MS, there are only a limited number of publications, where these methods of non-drug rehabilitation would be compared in a randomized controlled trial (Oken BS. 2004, Razazian N.2016, Garrett M. 2013, ).

At the same time, it is obvious that the correct comparison of these methods is of great interest for both researchers and specialists in the rehabilitation of patients with MS. This approach can expand our understanding of the mechanisms of functional disorders during MS and the ways to correct them. As a result, specialists will be able to combine the advantages of different approaches, improving the quality of rehabilitation.

It should also be noted, that contrary to the well-defined physical exercises protocols (Motl RW. 2020, Halabchi F. 2017), in most of the available at the moment studies, there is no detailed description for the performance of yoga protocols, i.e. those complexes of asanas, (special physical exercises), pranayama (breathing exercises) and techniques of mental relaxation, which were offered to patients, as well as the ways of their implementation. We can find an exception only in some articles, where the images and names of poses and the sequence of their performance are given (Guner S. 2015, Kishiyama S. 2002, de Oliveira G 2016, Najafı P. 2017). In other articles, the method of exercise and the use of yoga techniques 
are described only in general terms, such as hatha yoga, classical yoga, pranayama, yoga poses, breathing exercises. Typically, the frequency and duration of regular classes, the total number of classes, and the period of research are also specified.

However, our experience of teaching yoga, both in regular classes and for students with specific health problems, including patients with MS shows that for each particular disease or symptom, yoga exercises should be performed according to specially developed techniques. At the same time, each component of this technique: the choice of a set of poses, sequence, duration, and correctness of their performance, plays a crucial role in achieving the goals and is extremely important for ensuring the reproduction, verification, and further practical use of the results.

Therefore, for this study we used B.K.S. lyengar method of yoga training, which is one of the most recognized, time-tested, and systematic methods of classical hatha yoga (lyengar B.K.S. 1966.). At the moment, the efficiency of lyengar yoga has been demonstrated for the treatment of different symptoms, common for patients with MS, such as chronical pain (Crow EM. 2015), depression and anxiety (Scott T. 2019) fatigue (Oken BS. 2004, Bower J. 2012). Our own experience of conducting lyengar yoga classes for the patients with MS shows, that specially designed complexes of asanas have a beneficial effect on the condition and quality of life of the participants.

In this regard, themain aim of this study is to examine the effect of lyengar yoga on symptoms (such as walking, balance difficulties) and quality of life in patients with MS, versus physical therapy (exercise therapy) and no exercise.

\section{Methods}

Patients.

This study involved adult patients with MS of both genders who had not performed regular physical exercises before. The patients were randomly assigned to 3 groups (yoga, physical therapy, or waiting list), patients from the waiting list had an opportunity to enter the yoga program after the end of the trial period. The observation group (1, 2 or 3$)$ was known to both the patient and the doctor, but it was blinded for the rater. Participants were randomly divided into the yoga, physical therapy, or no exercise group. After 12 weeks of regular exercises (or absence of them), the effect of yoga and physical therapy on the functional status and quality of life of patients were evaluated. The training programs were developed specifically for MS patients. The MS treatment was a part of the routine practice, as prescribed by the treating neurologist. In the event of an exacerbation of MS or for other safety-related reasons (for example, side effects of an exercise), the patients were excluded from the study. The patients signed informed consent and got a patient information leaflet.

Physical methods. 
The yoga and exercise classes were held twice a week, for 12 weeks. Each session lasted about 60-75 minutes. The classes were held in a specially equipped hall, using supporting materials, under the guidance of experienced trainers and a trainer assistant. All exercises were performed by the study participants together, in accordance with the detailed instructions of the trainer.

In the exercise group, the class consisted of three parts: 10-15 minutes - a warm-up, which included exercises on stretching of the main muscle groups, flexibility, in combination with breathing training; 2535 minutes - endurance training, muscle strength, coordination and balance exercises, aerobic exercises; 10-15 minutes - relaxing techniques in combination with breathing exercises.

The yoga group practiced a special complex consisting of 13 basic and 4 alternative poses described in Appendix 1 and Appendix 2. The complex is basic, universal for patients with MS and is designed to ensure safety, but at the same time give practitioners the opportunity to progress and improve their condition. Alternative, lighter versions of those poses that may cause difficulties were also suggested, and basic postures were performed using additional materials to ensure comfortable exercise. The names of poses and the basic principles of their implementation correspond to the classification given in B.K.S. lyengar's book "Clarification of Yoga" by lyengar.

During the exercises, special attention was paid not only to the time of performance and the correct location of body parts, but also to the correct actions on tension, stretching and relaxation of the muscles of synergists and antagonists, as well as on getting patients' feedback after practicing a posture, increasing awareness and sensitivity of their body and its parts during the exercises. The alteration of activity and rest cycles was also an extremely important condition in the sequence, so that participants didnot get tired during the lessons. This is why the sequence involved both more active and more regenerative poses, with alternatives to some postures offered to the participants when fatigue or tension occured during the main exercise sequences. Throughout the study,the participants were also provided with the illustrated brochures with detailed descriptions and photographic illustrations of the exercises performed (Appendix 2).

Data and statistical analysis.

The data was collected during two visits to the research center: before the study began and at the end of the 12-week follow-up period. The survey on the visits included a doctor's assessment of the EDSS (Expanded Disability Scale Status), the SF-36 quality of life questionnaire, the fatigue scale, the Berg balance scale, the 6-minute walking test.

Descriptive statistics were used to describe demographics and all the data collected in the course of the study. For interval variables, the number of patients for each variable was calculated, the average arithmetic (with a 95\% confidence interval on the average, where applicable, and calculating the absolute change relative to the original value), standard deviation, median, minimum and maximum values. For one-order variables, the values of the median, 25 - and 75 percent were calculated. For nominal variables, the frequency of categories and confidence intervals for frequencies $(95 \%$ confidence intervals by the 
Klopper-Pearson method) were calculated for each visit in the course of the study. The number of values missed was specified for each variable.

It was planned to use statistical analysis methods, including adjustment with account of the plurality of comparisons. When other methods of statistical analysis were necessary, an additional adjustment to the Bonferroni method was introduced and substantiated.

Statistical analysis was conducted using SPSS/PASW Statistics, SPSS Inc.

\section{Results}

The distribution of study participants is presented below (Figure 1). In total, 80 people contacted the organizers of the study. Of these, 61 people were screened and 56 patients with MS were included in the study: 26 in the yoga group, 16 in the PT group and 14 in the waiting list. The remaining 5 people had no neurological symptoms of MS and therefore were not included in the study.

20 patients (11 in the yoga group, 7 in the PT group, and 2 in the waiting list) dropped out before passing the second test (the reasons for dropping out are shown in the table below). The most frequent reasons for dropping out of the yoga group were exacerbation of MS and non-compliance with the protocol, in the PT group - non-compliance with the protocol; 2 patients of the third group dropped out at their own request.

The number and the proportion of patients who successfully participated to the end of the study was 15 $(57.7 \%)$ in the yoga group, 9 (56.3\%) in the PT group and 12 (85.7\%) in waiting list ( $p=0.157$, Fisher's exact test).

According to the protocol, the endpoint analysis was carried out regarding the patients who had undergone the first and second examination (Per Protocol, PP). The number of the analyzed patients was 36 (15 in the first group, 9 in the second group and 12 in the third group). The results of the scale assessment are presented in the Table 1. Of these, 30 werewomen and 6 were men. The number and proportion (\%) of women in the groups was the following: 10/15 (66.7\%) in the yoga group, $9 / 9(100 \%)$ in the PT group and $11 / 12(91.7 \%)$ in the waiting list.The average age of patients (average \pm standard deviation) was $39 \pm 10.4$ years in the yoga group, $46.1 \pm 11.3$ years in the exercise group (PT) and $46.2 \pm 10.4$ years in the no-exercise group (waiting list); the difference between the averages in the study groups is statistically insignificant (by Student's t-test: (t-test value and df-value) 0,011023 and 40; 0,477188 and $28 ; 0,010097$ and 38 ). The average MS duration prior to the study was $12.6 \pm 8.4$ in the yoga group, $18.1 \pm 12.3$ in the exercise group and $18.5 \pm 7.9$ in the no exercise group. There were 14 patients with remitting MS, 1 patient with secondary-progressive MS in the yoga group; 6 patients with remitting MS, 2 patients with secondary-progressive MS and 1 patient with primary-progressive MS in the PT group; 7 patients with remitting MS, 5 patients with secondary-progressive MS in the waiting list. At the time of inclusion in the study, 15 (100\%) patients in the yoga group, 5 (55.6\%) patients in the PT group and 8 $(66.7 \%)$ patients in the waiting list were taking DMT. The most commonly taken drug was glatyramer 
acetate. In second place in frequency was interferon beta-1b. According to thesurvey on previously done types of exercise, most often, the patients exercised at home or just walked. However, nobody did exercise regularly for rehabilitation purposes. The median of EDSS was 4 points, the same in the yoga and PT groups and 4,5 points in the waiting list group.

Table 1

The results of the scale assessment are presented in the table

\begin{tabular}{|lllllll|}
\hline & $\mathbf{n}$ & $\begin{array}{l}\text { Sex } \\
(\mathbf{w} / \mathbf{m})\end{array}$ & Age & RRMS/PMS & $\begin{array}{l}\text { MS } \\
\text { duration }\end{array}$ & $\begin{array}{l}\text { EDSS, median and min, } \\
\text { max }\end{array}$ \\
\hline Yoga & 15 & $10 / 5$ & $39 \pm 10.4$ & $14 / 1$ & $12.6 \pm 8.4$ & $4[2-6]$ \\
\hline Exercise & 9 & $9 / 0$ & $46.1 \pm 11.3$ & $6 / 3$ & $18.1 \pm 12.3$ & $4[2-6,5]$ \\
$\begin{array}{l}\text { Waiting } \\
\text { list }\end{array}$ & 12 & $11 / 1$ & $46.2 \pm 10.4$ & $7 / 5$ & $18.5 \pm 7.9$ & $4,5[2,5-6,0]$ \\
\hline
\end{tabular}

There was no statistically significant difference between the groups in terms of improvement in MS symptoms as measured by the balance, walking test and fatigue scales. In the analysis of 8 criteria of SF-36 quality-of-life questionnaire by the covariation analysis (assessment of the influence of the group factor based on time dynamics, Time*Group) statistically significant differences were found in favor of the yoga group in terms of physical functioning (PF) ( $p=0.003)$, life activity (VT) $(p<0.001)$, mental health $(\mathrm{MH})(p=013)$, social functioning (SF) $(p=0.028)$ (Table 2). 
Table 2

In the analysis of 8 criteria of SF-36 quality-of-life questionnaire by the covariation analysis (assessment of the influence of the group factor based on time dynamics, Time* Group) statistically significant differences were found in favor of the yoga group in terms of physical functioning (PF) $(p=0.003)$, life activity $(V T)(p<0.001)$, mental health $(\mathrm{MH})(\mathrm{p}=013)$, social functioning (SF) $(p=0.028)$

\begin{tabular}{|c|c|c|c|c|}
\hline Outcome measure & Visit & Yoga & Exercise & Waiting list \\
\hline \multirow[t]{2}{*}{ Berg balance test } & Baseline & $48,0 \pm 8,9$ & $45,9 \pm 9,1$ & $46,6 \pm 6,8$ \\
\hline & 12 week & $50,5 \pm 5,2$ & $46,2 \pm 10,7$ & $49,0 \pm 6,3$ \\
\hline \multirow[t]{2}{*}{6 minute walking test } & Baseline & $451,9 \pm 119,9$ & $371,2 \pm 167,3$ & $379,0 \pm 163,0$ \\
\hline & 12 week & $469,5 \pm 142,9$ & $392,3 \pm 202,1$ & $386,3 \pm 158,2$ \\
\hline \multirow[t]{2}{*}{ Fatigue scale } & Baseline & $31,7 \pm 6,6$ & $29,0 \pm 6,8$ & $29,4 \pm 7,6$ \\
\hline & 12 week & $28,4 \pm 4,9$ & $27,1 \pm 6,2$ & $28,8 \pm 4,4$ \\
\hline \multicolumn{5}{|l|}{ SF-36 } \\
\hline \multirow[t]{2}{*}{ Physical Functioning } & Baseline & $57,14 \pm 25,624$ & $51,67 \pm 23,049$ & $57,08 \pm 18,885$ \\
\hline & 12 week & $70,00 \pm 20,381$ ** & $43,33 \pm 21,937$ & $51,67 \pm 25,346$ \\
\hline \multirow[t]{2}{*}{ Role-Physical Functioning } & Baseline & $28,33 \pm 36,433$ & $38,89 \pm 43,501$ & $20,83 \pm 31,683$ \\
\hline & 12 week & $41,67 \pm 37,401$ & $25,00 \pm 33,072$ & $25,00 \pm 28,204$ \\
\hline \multirow[t]{2}{*}{ Role-Emotional Functioning } & Baseline & $33,333 \pm 37,8028$ & $62,956 \pm 38,8988$ & $44,442 \pm 41,0362$ \\
\hline & 12 week & $55,560 \pm 44,8425$ & $62,967 \pm 42,3135$ & $44,450 \pm 32,8377$ \\
\hline \multirow[t]{2}{*}{ Vitality } & Baseline & $41,43 \pm 15,495$ & $45,63 \pm 19,353$ & $48,75 \pm 20,574$ \\
\hline & 12 week & $61,79 \pm 11,866^{\star \star \star}$ & $49,38 \pm 13,479$ & $43,33 \pm 17,233$ \\
\hline \multirow[t]{2}{*}{ Bodily pain } & Baseline & $70,833 \pm 27,3807$ & $82,500 \pm 15,8607$ & $62,292 \pm 26,3598$ \\
\hline & 12 week & $75,000 \pm 24,3120$ & $80,833 \pm 14,0312$ & $56,667 \pm 24,9621$ \\
\hline \multirow[t]{2}{*}{ General Health } & Baseline & $45,71 \pm 17,744$ & $43,89 \pm 8,580$ & $44,17 \pm 16,353$ \\
\hline & 12 week & $53,57 \pm 11,673$ & $44,44 \pm 17,756$ & $46,25 \pm 14,790$ \\
\hline \multirow[t]{2}{*}{ Social Functioning } & Baseline & $49,167 \pm 21,8899$ & $59,722 \pm 22,3413$ & $56,250 \pm 16,4282$ \\
\hline & 12 week & $75,833 \pm 18,5806^{*}$ & $63,889 \pm 26,1041$ & $61,458 \pm 23,5116$ \\
\hline \multirow[t]{2}{*}{ Mental Health } & Baseline & $50,00 \pm 16,585$ & $60,50 \pm 22,162$ & $55,67 \pm 21,334$ \\
\hline & 12 week & $68,29 \pm 12,982^{\star}$ & $59,00 \pm 25,545$ & $57,00 \pm 14,078$ \\
\hline
\end{tabular}

$*_{-} p<0.05$

$\star \star_{-} p<0.01$ 
$\star \star \star *-p<0.001$

In the course of the study, only 2 patients with UP were registered as having: pain (without clarification) and back pain, both cases in the PT group. There were no side effects during the yoga classes.

\section{Discussion}

This study has several unambiguous advantages: the group under research of non-drug rehabilitation (yoga) was compared not only with the control group without exercise, but also with the group treated by the standard method - PT. The sufficient duration of observation (12 weeks) was planned to assess the effect. The focus group may not be fully representative of the general population of patients with MS, but it is close to the population, which is treated by the researched methods (yoga, PT) in routine practice. In addition, there were no demographic restrictions on inclusion in the study. In general, the plan of the study corresponded to its' goals. The main limitations of the plan of this study can be attributed to the relatively small sample size, which reduces the power of the statistical tests. In addition, the planned number of patients (75) was not recruited for organizational reasons, including the introduction of restrictions in connection with the COVID-19 pandemic.

\section{Conclusions}

Regular yoga classes under the guidance of qualified staff are a promising method of non-drug rehabilitation of patients with MS with motor disorders. More research is needed to examine the impact of yoga on clinical patient improvement and quality of life indicators.

\section{Abbreviations}

CNS: Central nervous system;

DMT: Disease modifying therapy);

EDSS: Expanded Disability Scale Status;

MS: Multiple sclerosis;

PMS: Progressive MS;

PT: Physical therapy;

RRMS: Relapsing remitting MS;

UP: Undesirable phenomenon.

\section{Declarations}




\section{Ethics approval and consent to participate}

All experimental protocols were approved by a Buyanov City Hospital local ethics committee, (26, Bakinskaya Street, 115516, Moscow; +7 (0)495 322 17 10; gkb12@zdrav.mos.ru), ref: PC-01-18. The date of approval of the study protocol is 19/03/2017.

The informed consent was obtained from all participants of the study.

All methods were carried out in accordance with relevant guidelines and regulations.

\section{Consent for publication}

All participants completed and signed the informed consent form with a consent for publication.

\section{Availability of data and materials}

The datasets generated during and/or analysed during the current study are/will be available upon request from Elena Lysogorskaia (kutakovaev@gmail.com).

\section{Competing interests}

The authors declare that they have no competing interests.

\section{Funding}

The public noncommercial organization Yoga Practika Federation (Russia) funded the study. Yoga Practika Federation (Russia) payed the rent of yoga and PT rooms and payed PT and instructor of yoga for their work with MS patients during the project.

\section{Authors' contributions}

EL performed the neurological examination (blinded rater).

TI was the methodist and PT.

EU was the Methodist and instructor of yoga.

AM abd EL made a major contribution to writing the article.

MY and LB managed the project and made a contribution to writing the article.

\section{Acknowledgements}

We would like to thank Natalia Zryacheva, the vice-president of the Russian Society of Patients with Multiple Sclerosis, for her invaluable help in communicating with the community of MS patients and coordinating the study participants. 


\section{References}

1. Skierlo S, Rommer PS, Zettl UK. Symptomatic treatment in multiplesclerosis-interim analysis of a nationwide registry. Acta Neurol Scand. 2017; doi:10.1111/ane.12612.

2. Rogers KA, MacDonald M. Therapeutic Yoga: Symptom Management for Multiple Sclerosis. J Altern Complement Med. 2015; doi:10.1089/acm.2015.0015

3. Khan F, Turner-Stokes L, Ng L, Kilpatrick T, Amatya B. Multidisciplinary rehabilitation for adults with multiple sclerosis. Cochrane Database of Systematic Reviews 2007; doi:10.1002/14651858.CD006036.pub2

4. Alphonsus KB, Su Y, Arcy CD. The effect of exercise, yoga and physiotherapy on the quality of life of people with multiple sclerosis: Systematic review and meta-analysis. Complement Ther Med. 2019; doi:10.1016/j.ctim.2019.02.010.

5. Motl RW. Exercise and Multiple Sclerosis. Adv Exp Med Biol. 2020;1228:333-343. doi: 10.1007/978981-15-1792-1_22.

6. Miller P, Soundy A. The Pharmacological and Non-Pharmacological Interventions for the of Fatigue Related Multiple Sclerosis. J Neurol Sci. 2017; doi:10.1016/j.jns.2017.08.012.

7. Pilutti LA, Platta ME, Motl RW, Latimer-Cheung AE. The safety of exercise training in multiple sclerosis: a systematic review. J Neurol Sci. 2014; doi:10.1016/j.jns.2014.05.016

8. Wens I, Dalgas U, Vandenabeele F, Grevendonk L, Verboven K, Hansen D, Eijnde BO. High Intensity Exercise in Multiple Sclerosis: Effects on Muscle Contractile Characteristics and Exercise Capacity, a Randomised Controlled Trial. PLoS One. 2015 Sep 29;10(9):e0133697. doi:

10.1371/journal.pone.0133697

9. Razazian N, Yavari Z, Farnia V, Azizi A, Kordavani L, Bahmani DS et al. Exercising Impacts on Fatigue, Depression, and Paresthesia in Female Patients with Multiple Sclerosis. Med Sci Sports Exerc. 2016; doi:10.1249/MSS.0000000000000834.

10. Heine M, van de Port I, Rietberg MB, van Wegen EE, Kwakkel G. Exercise therapy for fatigue in multiple sclerosis. Cochrane Database Syst Rev. 2015; doi:10.1002/14651858.CD009956.pub2.

11. Patil NJ, Nagaratna R, Garner C, et al. Effect of integrated yoga on neurogenic bladder dysfunction in patients with multiple sclerosis: a prospective observational case series. Complement Ther Med. 2012;20:424-430

12. Salgado BC, Jones M, Ilgun S, et al.. Effects of a 4-month Ananda yoga program on physical and mental health outcomes for persons with multiple sclerosis. Int J Yoga Therap. 2013;23:27-38

13. Karbandi S, Gorji MA, Mazloum SR, Norian A, Aghaei N. Effectiveness of Group Versus Individual Yoga Exercises on Fatigue of Patients with Multiple Sclerosis. N Am J Med Sci. 2015; doi:10.4103/1947-2714.159332

14. Chobe S, Bhargav H, Raghuram N, Garner C. Effect of integrated Yoga and Physical therapy on audiovisual reaction time, anxiety and depression in patients with chronic multiple sclerosis: a pilot study. J Complement Integr Med. 2016 Sep 1;13(3):301-309. doi: 10.1515/jcim-2015-0105 
15. Cohen ET, Kietrys D, Fogerite SG, et al. Feasibility and Impact of an 8-Week Integrative Yoga Program in People with Moderate Multiple Sclerosis-Related Disability: A Pilot Study. Int J MS Care. 2017; doi:10.7224/1537-2073.2015-046

16. Kahraman T, Ozdogar AT, Yigit P, et al. Feasibility of a 6-Month Yoga Program to Improve the Physical and Psychosocial Status of Persons with Multiple Sclerosis and their Family Members. Explore (NY). 2018; doi:10.1016/j.explore.2017.07.006

17. Nejati S, Esfahani SR, Rahmani S, Afrookhteh G, Hoveida S. The Effect of Group Mindfulness-based Stress Reduction and Consciousness Yoga Program on Quality of Life and Fatigue Severity in Patients with MS. J Caring Sci. 2016 Dec 1;5(4):325-335. doi: 10.15171/jcs.2016.034.

18. Oken BS, Kishiyama S, Zajdel D, Bourdette D, Carlsen J, Haas M, Hugos C, Kraemer DF, Lawrence J, Mass M. Randomized controlled trial of yoga and exercise in multiple sclerosis. Neurology. 2004 Jun 8; 62(11):2058-64. doi: 10.1212/01.wnl.0000129534.88602.5c.

19. Garrett M, Hogan N, Larkin A, Saunders J, Jakeman P, Coote S. Exercise in the community for people with minimal gait impairment due to MS: an assessor-blind randomized controlled trial. Mult Scler. 2013 May;19(6):782-9. doi: 10.1177/1352458512461966.

20. Halabchi F, Alizadeh Z, Sahraian MA, Abolhasani M. Exercise prescription for patients with multiple sclerosis; potential benefits and practical recommendations. BMC Neurol. 2017 Sep 16;17(1):185. doi: 10.1186/s12883-017-0960-9

21. Guner S, Inanici F.Yoga therapy and ambulatory multiple sclerosis Assessment of gait analysis parameters, fatigue and balance. J Bodyw Mov Ther. 2015; doi:10.1016/j.jbmt.2014.04.004.

22. Kishiyama S, Carlsen J, Lawrence J, Small E, Zajdel D, Oken B. Yoga as an Experimental Intervention for Cognition in Multiple Sclerosis. International journal of yogatherapy. 2002; 12. 57-62.

23. de Oliveira G, Tavares M da C, de Faria Oliveira JD, Rodrigues MR, Santaella DF. Yoga Training Has Positive Effects on Postural Balance and Its Influence on Activities of Daily Living in People with Multiple Sclerosis: A Pilot Study. 2016; doi:10.1016/j.explore.2016.06.005.

24. Najafi P, Moghadasi M. The Effect of Yoga Training on Enhancement of Adrenocorticotropic Hormone (ACTH) and Cortisol Levels in Female Patients With Multiple Sclerosis. Complement Ther Clin Pract. 2017; doi:10.1016/j.ctcp.2016.11.006.

25. lyengar B.K.S. Light on Yoga. New York: Schocken Books; 1966.

26. Crow EM, Jeannot E, Trewhela A. Effectiveness of lyengar yoga in treating spinal (back and neck) pain: A systematic review. Int J Yoga. 2015 Jan; 8(1):3-14. doi: 10.4103/0973-6131.146046.

27. Scott T, Gerbarg P, Silveri M, Nielsen G, Owen L, Nyer M, Brown R, Streeter C. Psychological Function, lyengar Yoga, and Coherent Breathing: A Randomized Controlled Dosing Study. J Psychiatr Pract. 2019, Nov; 25(6):437-450. doi: 10.1097/PRA.0000000000000435.

28. Bower J, Garet D, Sternlieb B, Ganz P, Irwin M, Olmstead R, Greendale G. Yoga for persistent fatigue in breast cancer survivors: a randomized controlled trial. Cancer. 2012 Aug 1; 118(15):3766-75. doi: 10.1002/cncr.26702. 


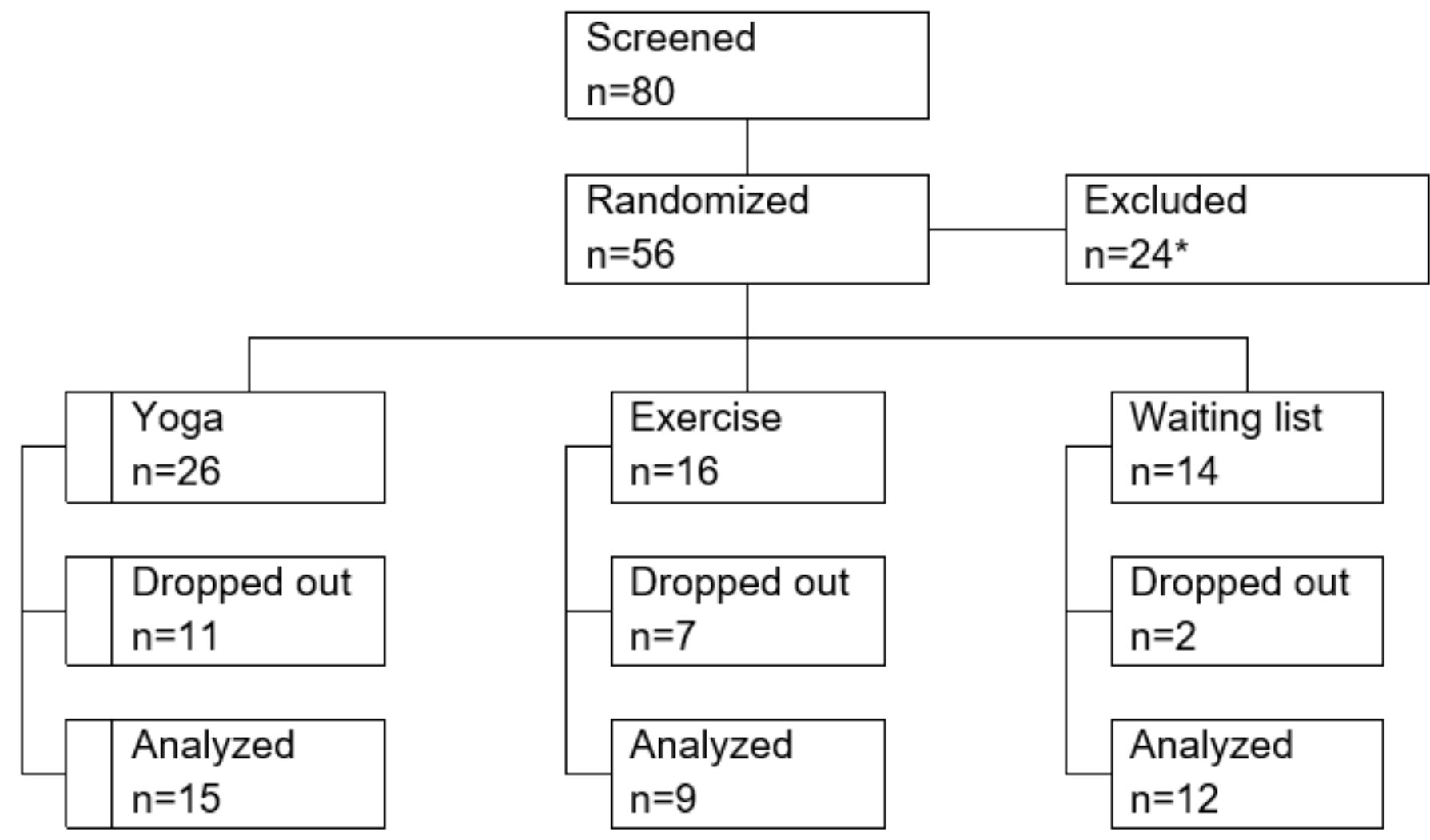

Figure 1

*- 5 patients didn't meet inclusion/exclusion criteria, 12 declined to participate, 7 other reasons.

\section{Supplementary Files}

This is a list of supplementary files associated with this preprint. Click to download.

- Appendix1.docx 